\title{
Intermédialités
}

Histoire et théorie des arts, des lettres et des techniques

Intermediality

History and Theory of the Arts, Literature and Technologies

\section{Colonel Chabert ou le revenant intempestif}

\section{Alain Brossat}

Numéro 10, automne 2007

Disparaître

Disappearing

URI : https://id.erudit.org/iderudit/1005553ar

DOI : https://doi.org/10.7202/1005553ar

Aller au sommaire du numéro

Éditeur(s)

Centre de recherche sur l'intermédialité

ISSN

1705-8546 (imprimé)

1920-3136 (numérique)

Découvrir la revue

Citer cet article

Brossat, A. (2007). Colonel Chabert ou le revenant intempestif. Intermédialités /

Intermediality, (10), 61-75. https://doi.org/10.7202/1005553ar
Résumé de l'article

En 1832, Balzac compose une longue nouvelle, Le Colonel Chabert. Il y imagine le destin peu commun de cet officier de l'armée impériale qui, enseveli sous un tas de cadavres à la bataille d'Eylau, passe pour mort auprès de ses compagnons et de ses proches. Il échoue ensuite sans fin à faire reconnaître ses titres, comme être vivant parmi les vivants, tout simplement - une personne humaine. Et si, donc, Le Colonel Chabert était le premier grand texte littéraire moderne consacré à la question de la disparition? 


\title{
Colonel Chabert ou le revenant intempestif
}

\author{
Alain Brossat
}

- $n$ 1832, Balzac publie cette longue nouvelle qui s’appelle Le Colonel Chabert.

- On est donc au début de la Monarchie de Juillet, après le long épisode répressif et obscurantiste, peuplé par les spectres de l'aventure napoléonienne, de la Restauration. Au début du récit, après quelques préliminaires (tableau d'ambiance d'une étude d'avoué dans le centre de Paris), on assiste à cette scène qui nous conduit au cœur du sujet. Un vieillard d'apparence misérable se présente à l'étude de l'avoué Derville et demande à parler à celui-ci. L'avoué n'est pas là et, mi-sérieux, mi-moqueur, son clerc conseille au vieil homme de revenir à une heure du matin, lui disant que ce n'est qu'au milieu de la nuit que l'on peut espérer trouver son maître qui est toujours très occupé. Il espère ainsi se débarrasser de ce fâcheux. Mais à l'heure dite, l'homme est à nouveau là, avec son allure de spectre glacé. Et c'est alors qu'a lieu cet échange qui nous immerge dans notre sujet:

- Monsieur, lui dit Derville, à qui ai-je l'honneur de parler?

- Au colonel Chabert?

- Lequel?

- Celui qui est mort à Eylau, répondit le vieillard.

- En entendant cette singulière phrase, le clerc et l'avoué se jetèrent un regard qui signifiait: «c'est un fou ${ }^{1}$ ! (CC, p. 43).

La scène se passe en 1818, soit trois ans après la chute de l'Empire.

Contrairement à ce qu'on pourrait imaginer, Balzac n'est pas ici en train de s'essayer au genre de la littérature noire ou fantastique (tale of terror en anglais) façon Mystères du château d'Udolphe d'Ann Radcliffe ou Melmoth de Charles

1. Honoré de Balzac, Le colonel Chabert [1835], Monaco, Éditions du Rocher, coll.«Les grands classiques», 1994. Désormais, les références à cet ouvrage seront indiquées par le sigle «CC» suivi de la page et placées entre parenthèses dans le corps du texte. 
Mathurin, il est en train d'écrire un des premiers grands textes modernes qui empoignent la question de la disparition. Ou peut-être pourrait-on dire les choses autrement: Le colonel Chabert est un texte où l'on voit Balzac et la littérature saisis par la question de la disparition, dans son temps où celle-ci impose, déjà, son actualité.

Reprenons donc pour essayer de donner un sens à cette formule violente qui cultive le paradoxe - «celui qui est mort à Eylau» - , prononcée par celui-là même qui est supposé être le mort. Chabert fait partie de cette petite noblesse d'Empire promue par le métier des armes, récompensée pour ses bons et loyaux services par des biens et des terres, mais constamment exposée aux aléas de la guerre; ces gens-là ne sont pas des soldats d'opérette, mais des guerriers endurcis qui portent les armes de l'Empire jusque sous les murs de Moscou. Et c'est ainsi que Chabert, pris dans une charge de la cavalerie russe à la bataille d'Eylau (Prusse orientale, 1807), est jeté dans une fosse commune, tenu pour mort, à l'issu de ce carnage qui fit - et cela ne relève pas de l'imagination de Balzac, mais des annales historiques - dans les 40000 morts. Ce chiffre considérable, mais assez courant dans le registre des hécatombes napoléoniennes, est à retenir, il n'est pas sans importance pour la suite.

Seulement voilà, Chabert n'est pas mort, il n’a été qu’assommé et blessé par un coup de sabre russe. Il revient donc à lui et s'extrait d'entre les morts (l'image lazaréenne), en s'accrochant, nous dit-il, au bras d'un cadavre, «un Hercule» (cC, p. 47). Il est ensuite recueilli et soigné par des humbles, des paysans allemands, puis admis dans un hôpital où il guérit lentement. Très tôt, il comprend ce que sa situation a de litigieux, c'est-à-dire à quelles difficultés expose la particularité d'être revenu d'entre les morts: dans la ville allemande où il a été soigné, donc, Heilsberg, il fait constater «dans les formes juridiques voulues par le droit du pays la manière miraculeuse dont [il est] sorti de la fosse des morts» (CC, p. 49). Intéressant est le biais juridique adopté d'emblée ici : «revenir d'entre les morts» n'est pas, selon cette approche, en premier lieu un problème métaphysique ou religieux, en dépit de l'emploi du mot «miraculeux», ce n'est pas même un problème existentiel ou moral, c'est un problème de droit: il faut faire valoir son droit à vivre encore (à être réintégré dans la société, à retrouver ses droits de vivant), alors même qu'on a été rangé ou compté parmi les morts. Grande lucidité prémonitoire de Chabert, ici, qui saisit que les vivants ont horreur des re-venants, pas seulement en tant que spectres ou fantômes, mais aussi et surtout, plus trivialement, parce que le propre des vivants, c'est de prospérer sur le compte des morts et d'occuper immédiatement les espaces laissés vacants par eux. Donc, en premier lieu, une question de droit: Chabert veut établir à toutes fins utiles ses 
titres à exister, en dépit de sa mort annoncée. Démarche ou précaution simple, en apparence, pure formalité; travail de Sisyphe, en vérité.

Chabert fait du Arendt sans le savoir; il a compris que pour retrouver une existence qualifiée, une vie sociale (de vivant parmi les vivants), il lui faut commencer par faire valoir, bizarrement, son droit à exister en dépit de la publicité de sa mort. Il ne suffit pas de la présence d'un corps, encore faut-il que du droit s'attache à cette présence, et pour que le droit prenne corps, il faut que le corps fasse corps avec une identité définie. Il faut que Chabert puisse dire non seulement: «voilà, je suis un survivant de la bataille d'Eylau », mais bien : «je suis Chabert, oui, ce Chabert-là et nul autre, et je vous le prouve»!

Première démonstration requise, préliminaire à toute ouverture de droit(s), c'est-à-dire à toute validation d'une vie a priori indéterminée en tant qu'elle est identifiée comme celle d'une personne humaine nommable, une singularité: si vous ne pouvez pas répondre de façon probante dans les formes requises, à la question «qui es-tu? », ou si vous ne pouvez pas présenter les titres attendus à l'appui de votre réponse, vous n'existez pas. Plus l'on se rapproche de nos formes de vie modernes et de ce qui les appareille, et plus le niveau d'exigence s'élève, concernant la réponse au «qui? » et concernant les documents et preuves à l'appui. Rappelez-vous, par contraste, la grande facilité avec laquelle, Ulysse, tout au long de son long voyage, abuse ses interlocuteurs à toutes fins utiles quand on lui demande qui il est. Ulysse vit dans un monde où l'on ne demande pas leurs «papiers » aux gens; Chabert si, déjà.

Et voilà, donc: la guerre reprend, Chabert est chassé, en tant qu'ennemi de la ville où il a été soigné, il ne peut pas emporter avec lui les titres qui établissent son identité. Commence alors pour lui une vie errante, ballottée par les événements de la guerre, une existence acosmique (Arendt ${ }^{2}$ ) ou, comme il le dit, de vagabond, un Charlot napoléonien. Il est contraint de mendier son pain et se trouve finalement emprisonné pendant deux ans à l'étranger, comme suspect. Et c'est là que prend forme à proprement parler son calvaire. Pourquoi? Parce qu'il est incapable de répondre de façon probante à la question: "Qui es-tu?». Qui lui pose cette question? Des autorités, des policiers, prompts à voir des espions ou des suspects partout, dans cette Europe moyenne en guerre. Et quand il répond: «je suis le colonel Chabert», ces autorités lui disent: «Prouve-le! Où sont les papiers qui l'attestent?» Et comme il ne peut pas le prouver, on l'enferme et on

2. Hannah Arendt, «Charlie Chaplin : le suspect», dans La Tradition cachée: le juif comme paria, trad. Sylvie Courtine-Denamy, Paris, Éditions Christian Bourgois, coll. «Choix essais», 1987, [1948]. 
le prend soit pour un individu dangereux (version policière), soit pour un fou (version compassionnelle, populaire). Je le cite:

Après deux ans de détention que je fus obligé de subir, après avoir entendu mille fois mes gardiens disant: «voilà un pauvre homme qui croit être le colonel Chabert! » à des gens qui répondaient: «le pauvre homme! », je fus convaincu de l'impossibilité de ma propre aventure, je devins triste, résigné, tranquille, et renonçai à me dire le colonel Chabert afin de pouvoir sortir de prison et revoir la France. (CC, p. 50).

Ce passage est important, puisqu'il nous achemine lentement mais sûrement vers la situation que Lyotard décrit au début du Différend3 : Chabert n’est pas encore exactement un «plaignant», ici, quoiqu'il subisse un tort très avéré, mais en tout cas, ce qu'il demande en l'occurrence, ce n'est pas une réparation, 64 c'est simplement à être validé, reconnu pour ce qu'il sait être. Ce qui est déjà totalement inscrit dans la problématique lyotardienne, c'est l'enjeu du défaut de preuve. Ici, ce contraste déchirant entre ce que je sais le mieux - qui je suis, du moins, du point de vue de mon identité, mon nom, ma «naissance » - et mon impuissance à faire partager cette évidence par d'autres, à passer d'un régime du «je » à un régime du «nous». C'est la même configuration (toutes choses égales par ailleurs) que celle du survivant qui, mieux que quiconque, sait que $99 \%$ de ceux qui ont débarqué avec lui du convoi sur la rampe d’Auschwitz sont morts (il les a vus entrer dans la chambre à gaz), mais est dans l'incapacité d'en apporter la preuve formelle à un public incrédule, blasé ou sceptique.

On retrouve cette figure du pur déchirement, cette miniature du désastre, dans une scène terrible du film Le temps du loup (2003) de Michaël Haneke. On y voit une femme et ses enfants, dans un groupe de réfugiés, en un temps incertain de guerre mal identifiée (guerre civile, guerre ethnique, désastre nucléaire...) reconnaître ou croire reconnaître tout à coup un des membres d'une bande qui, au début du film, a froidement assassiné son mari pour les dépouiller. La femme et les enfants, crient, jurent, adjurent les quelques hommes en armes qui occupent la place du «juge», dans cette scène, d'arrêter cet assassin, mais celui-ci ne se démonte pas, il n’a jamais vu ces gens-là, dit-il, il n’a jamais été à l'endroit qu'elle dit, et il se défend si bien qu'à la fin, le spectateur ne sait plus, pas davantage que les hommes en armes qui renvoient les parties dos-à-dos: on ne peut pas juger quelqu'un sur la foi d'un seul «témoignage»...

Ce qui est à noter, dans la situation de Chabert, c'est la forme que prend la chute de celui qui ne peut faire valoir son propre récit à propos de ce qu'il

3. Jean-François Lyotard, Le Différend, Paris, Les Éditions de Minuit, coll. «Critique », 1983 , p. 16 et sq. 
est auprès de la société - je suis Chabert —, c'est-à-dire faire coïncider ce qu'il énonce avec la réalité ; la forme que revêt cette chute est double et particulièrement draconienne - ce que j'appelle un désastre intime: soit il sera traité comme un criminel, soit comme un fou. Dans le premier cas, on va considérer qu'il ment, qu'il est un imposteur et donc que son mensonge recouvre quelque crime ou intention d'en commettre; dans le second, on verra dans son énoncé la manifestation d'un délire. Et donc, à défaut de pouvoir faire coïncider son énoncé et la réalité reconnue comme telle par tous les autres, il va devoir se plier à cette évidence absolument catastrophique: qu'il n'est aucune forme de réalité humaine, aussi simple, aussi évidente, aussi immédiate soit-elle pour un sujet donné (individuel ou collectif), qui ait la capacité de s'imposer par elle-même, sans passer par des procédures de validation, d'identification, de reconnaissance sociales, politiques, juridiques. Ce qui pourrait se dire autrement, au prix d'un léger déplacement: il n’est aucune vérité (vérité d'expérience, vérité d'évidence perceptive, etc.) dont un individu est le siège qui tienne face au doute ou aux certitudes adverses de l'opinion et de l'autorité coalisées. Et donc, pour revenir à la vie, et dans l'espoir de faire valoir ses droits ultérieurement, Chabert va cesser de se dire Chabert, c'est-à-dire soumettre son récit aux conditions de la tyrannie de l'opinion, entrer dans la peau du «pauvre» fou.

Résumons donc cette première «leçon» que nous dispense ici Balzac: il n'est aucune espèce de réalité, aussi élémentaire ou massive soit-elle, qui soit assurée de faire l'objet d'un récit assuré, découlant d'elle-même comme de source. En d'autres termes, il ne suffit pas d'«y avoir été», de l'avoir «vu de ses propres yeux», de l'avoir «soi-même subi» pour pouvoir composer un récit. Et plus il est question de s'établir comme le narrateur s'inscrit dans l'horizon des faits ou épreuves «hors normes », rares ou «extrêmes» et plus les tentatives de mettre en récit seront exposées à tous les périls. Le problème de Chabert est indissociable de la fosse commune, de la dimension exterminationniste des campagnes napoléoniennes; il ne se poserait pas si Chabert avait survécu, après avoir été tenu pour mort, à un accident de chasse.

Tel est donc le premier moment du désastre dont Chabert connaît l'épreuve en tant que survivant rejeté et stigmatisé ou, si l'on veut, innocent puni et devenant victime. Ici, des rapprochements avec des personnages de Kafka pourraient s'imposer, la petite musique théologique ou métaphysique en moins, et encore: quand le hasard de la survivance à un désastre devient une sorte de faute personnelle, on entre dans un domaine d'indétermination morale tout à fait singulier. C'est cela l'épreuve effroyable à laquelle doit faire face Chabert: être puni sans fin pour le crime imaginaire d'avoir survécu à un cataclysme. À l'évidence, faire 
face à une telle situation requiert des ressources morales plus importantes que celles que mobilise le courage guerrier. Il est plus facile d'être un brave ou un héros qui tombe au champ d'honneur (avec tous les autres) que d'être ce survivant qui voit le monde entier se liguer contre lui pour empêcher sa réapparition ou, ce qui revient au même, pour tramer sa disparition. Le cœur de cette épreuve, c'est ce «seul contre tous» sans fin, tel que l'endure Chabert.

C'est lorsque Chabert finit par rentrer en France, en pleine Restauration, donc, dans un monde où il fait partie, politiquement, des vaincus et des stigmatisés, où il est out of time, que se précise l'articulation du tort subi et de l'impossibilité de faire valoir une plainte. En effet, de retour dans son pays après cette longue odyssée (je reviendrai sur le rapprochement obligé avec les tribulations d'Ulysse), Chabert découvre que son épouse chérie, se pensant veuve, s'est empressée de se remarier avec un aristocrate d'ancienne facture, qu'elle est devenue comtesse Ferraud, du nom de son nouveau mari, et qu'elle a accaparé les trente mille livres de rente qui revenaient à Chabert. Naturellement, le retour d'outre-tombe du colonel ne fait pas du tout l'affaire de la donzelle et, lorsque notre homme a entrepris de faire valoir premièrement qu'elle n'avait pas cessé d'être sa femme, au plan légal, et secondement qu'elle avait capté son bien, elle l'a purement et simplement chassé comme un gueux et un imposteur - dont il a toute l'apparence - , ce qui tombe bien pour elle. Et donc, les choses en sont là quand se produit cette fameuse conversation, évoquée au début, entre Chabert et l'avoué Derville. Voici le résumé que fait le colonel, explicitement plaignant cette fois, de sa situation sans issue, demandant au juriste (les professions d'avoué et d'avocat sont mal distinguées à l'époque) de prendre en charge sa cause:

Mais, Monsieur, la comtesse Ferraud n'est-elle pas ma femme? Elle possède trente mille livres de rente qui m’appartiennent, et ne veut pas me donner deux liards. Quand je dis ces choses a des avoués; à des hommes de bon sens; quand je propose, moi, mendiant, de plaider contre un comte et une comtesse; quand je m'élève, moi, mort, contre un acte de décès, un acte de mariage et des actes de naissance, ils m'éconduisent, suivant leur caractère, soit avec cet air froidement poli que vous savez prendre pour vous débarrasser d'un malheureux, soit brutalement, en gens croyant rencontrer un intrigant ou un fou. (CC, p. 51)

Pour être reconnu ou enregistré comme plaignant, encore faut-il, dirait Arendt, que vous soyez constitué comme sujet juridique ou politique, c'est-à-dire que vous puissiez présenter vos titres d'appartenance. Or, ce n'est pas le cas de Chabert. Sa situation dans sa propre société, dans son propre pays, est ici exactement la même que celle du réfugié, de l'apatride, de celui qui a perdu tous ses droits et qui, simple corps, se trouve réduit à merci, exposé au bon vouloir ou à 
l'arbitraire des pouvoirs et des puissants. Il est, tout à la fois un sans papiers et un SDF (il vit dans une espèce de taudis qui horrifie l'avoué lorsque celui-ci s'y égare une fois), et, à ce titre, frappé d'inconsistance sociale, politique et juridique, ne pesant rien dans cet Ancien régime d'opérette qu'est la société de Louis XVIII et de Charles X, il n'a aucune chance de faire reconnaître le tort subi, ni enregistrer le litige qui l'oppose à son ancienne femme. Il est un invisible, un exclu de l'intérieur. Ces motifs nous sont devenus familiers du fait de la multiplication des cloaques et des lieux de relégation dans nos sociétés. Comme le plus souvent dans ces cas-là, la dimension sociale de l'acosmisme (un pauvre) se combine avec la dimension juridico-politique (un sans-papiers) pour composer la figure de celui qui ne peut accéder, selon la formule arendtienne convenue, au droit de faire valoir ses droits. Chabert décrit cette épreuve non pas comme exclusion ou mise au ban, mais comme enfouissement, ensevelissement: "J'ai été enterré sous des morts; mais maintenant, je suis enterré sous des vivants, sous des actes, sous des faits, sous la société toute entière, qui veut me faire rentrer sous terre! » (CC, p. 51). Cette figure de la désolation (solitudo) fait penser, toutes choses égales par ailleurs, à celle du musulman des camps nazis, telle que l'évoque Agamben commentant Primo Levi ${ }^{4}$ : même position indéterminée du sujet entre les vivants et les morts. Ni vivant qualifié (il a perdu son nom, il est «personne » et non une personne), ni mort, dans cet entre-deux fatal, sur cette ligne de fracture d'où il ne peut articuler un récit de légitimation, ni engager une procédure de validation de ses titres à être qui il est.

Aussi bien, sa situation s'apparente ici à celle de l'ancien détenu du goulag qui, dans la nouvelle de Vassili Grossman (l'auteur de l'immortel Vie et destin) Tout passe ${ }^{5}$, revient chez lui après la mort de Staline et la fin de la terreur de masse. Au lieu d'être accueilli en victime, il est traité en gêneur et en suspect, car son retour d'«entre les morts» (rappelons-nous le titre du témoignage de déportation de Dostoïevski, Souvenirs de la maison des morts) accuse la lâcheté et le silence de tous ceux qui ont échappé à la répression au prix de leurs petits et grands compromis avec l'ordre totalitaire. Après la «mort» concentrationnaire, une seconde mort attend le revenu-revenant du camp, cette sorte d'ostracisme rampant qui le frappe dans une société transie, rompue et déjetée par deux décennies de terreur et d'exterminations. Avec Chabert, il y a ce même effet

4. Giorgio Agamben, Ce qui reste d'Auschwitz: l'archive et le témoin, trad. de Pierre Alferi, Paris, Payot, coll. «Bibliothèque Rivages», 1999, p. 23 et sq.

5. Vassili Grossman, Tout passe, trad. Jacqueline Lafond, Paris, Julliard, coll. «LẦge d'homme», 1984 . 
de contretemps: la bonne société revancharde, parasitaire et obscurantiste de la Restauration ne supporte pas le retour de ce spectre qui, même en lambeaux, continue d'incarner l'esprit d'un temps d'héroïsme, de bruit et de fureur grandioses auquel elle tourne le dos.

Il y a un conflit ouvert entre cette société incarnée par la comtesse Ferraud, l'ex-épouse de Chabert, son (nouveau) mari, et le rescapé des temps napoléoniens, conflit à propos, tout simplement, de ce qu'est le réel: pour les premiers, le réel se réduit à ce qu'a imposé le cours des choses, il est ce qui l'a emporté en rien d'autre, il est donc, littéralement, le monde des vainqueurs, ce présent-là irrécusable, tel qu'il s'incarne dans des institutions, des carrières, des fortunes, des succès mondains, etc. Le réel, c'est l'advenu, tel qu'on l'a sous les yeux et tel qu'il coïncide ainsi avec le devant-être : s'il a l'allure qu'il a, c'est bien qu'il fallait qu'il en soit ainsi. Dire le réel est, dans cette optique, simple : il suffit de raconter ce qu'on a sous les yeux, c'est un réel de journaliste. Pour Chabert, au contraire, le réel, c'est cela même qui se dérobe au récit, c'est-à-dire cette guerre sourde entre d'autres possibles de l'histoire (Napoléon vainqueur à Waterloo et ce qu'on peut imaginer qui se serait enchaîné à cette victoire...), des épopées refoulées, des expériences condamnées au silence, des bifurcations biffées, et ce qui se montre, mais qui est l'inessentiel. Ce que met en scène, entre autres, l'obstination de Chabert à revenir, à faire retour dans la société française, c'est, bien évidemment, le conflit de deux aspirations à faire époque. Le «retour» réussi de Chabert signifierait infiniment davantage qu'un sauvetage individuel - une leçon d'histoire. Ce serait la démonstration que ce qui fait époque, en ce début du XIX siècle, en dépit des aléas politiques et militaires, ce n’est évidemment pas la restauration de pacotille, mais bien la séquence Révolution-Empire. D’où la haine et la crainte que suscitent le «spectre » Chabert auprès des parvenus du nouveau régime. Il est redouté et haï comme celui qui incarne la menace d'une démonstration de l'inconsistance de l'histoire des vainqueurs et du retour toujours possible d'une autre histoire, épique et plébéienne, en dépit de tout. Il faut donc qu’il disparaisse.

Et inversement, Chabert est écrasé par le fardeau de cette mission impossible: faire la démonstration de l'imposture des vainqueurs, faire valoir contre le cours des choses un récit qui redresse l'histoire, un récit qui débouche sur des actions de restitution, de réparation, sur une scène où la justice est rétablie: où le guerrier qui a payé de sa personne se voit rétabli dans ses droits, où les profiteurs et les parasites sont démasqués. Mais qui, par ses seules forces, pourrait prétendre redresser le récit de l'histoire pour faire valoir, seul contre tous, ce qui vraiment fait époque? Chabert, ici, par certains traits, fait penser au conspirateur Blanqui affrontant ses juges et incarnant envers et contre tout le mouvement infini de 
la révolution face à ces veilleurs de nuit ${ }^{6}$ : son époque, son actualité ne pourra jamais coïncider avec la leur, en dépit de leur coprésence, et c'est la raison pour laquelle il doit, lui aussi, disparaître dans un cul-de-basse-fosse.

Second résumé, donc: la possibilité de faire enregistrer une plainte est sans rapport avec l'énormité du tort subi. La première condition pour qu'une telle procédure puisse s'engager (et donc la possibilité d'articuler et de rendre audible un récit de plaignant), c'est que celui qui entend la formuler soit identifiable en tant qu'inscrit, inclus dans une sphère juridique. Sinon, son récit et sa plainte seront de l'ordre du flatus vocis, un bruit, une rumeur, un effet de voix qui se perdent dans l'éther et ne trouvent pas de répondant dans une situation d'interlocution. C'est bien ce que dit, presque littéralement, Chabert: il apparaît trop excentré ou trop insignifiant aux yeux de ceux auprès desquels il va chercher assistance pour être, tout bonnement, entendu. Derrière cette surdité de l'ordre social et politique à la plainte de cette variété de déclassé, se présente une possibilité redoutable - celle de son devenir-fou: le fait même d'avoir raison contre tous les autres et de persister à faire valoir son droit et sa vérité contre eux vous fait tomber dans la folie. La folie est ici un statut social, la condition propre de celui qui s'obstine à vouloir faire entendre son propre récit de vérité contre tous les autres.

En principe, d'ailleurs, faute de pouvoir faire exposer vos preuves et faire valoir votre bon droit, vous avez toutes les chances de glisser effectivement, soit vers la folie (des grandeurs, manie de la persécution, etc.), soit vers la criminalité. C'est le paradigme qu'expose magnifiquement la nouvelle de Kleist Michael Kohlhaas7. Constamment, la victime d'un tort extrême, quel qu'il soit (la violence génocidaire n'étant qu'un cas parmi d'autres) est exposée, dans le prolongement de son échec à produire un récit probant de ce tort, à redoubler son glissement hors du champ de la vie commune (et donc à donner raison à ceux qui demeurent incrédules ou méfiants face à son récit) en entrant dans la peau de ces personnages: le fou, le criminel, ou encore, le suicidé. A l'origine, Michael Kohlhaas est absolument dans son bon droit: il a subi une injustice, une spoliation, du fait de ce grand seigneur brutal et arrogant qui a volé et maltraité ses chevaux. Seulement voilà: rencontrant l'impossibilité de faire entendre sa plainte devant

6. Auguste Blanqui, Instructions pour une prise d'armes [1868]; L'éternité par les astres: hypothèse astronomique [1872] et autres textes, Paris, Société encyclopédique française, Éditions de la Tête de feuilles, coll. «Futur antérieur», 1973.

7. Heinrich von Kleist, Michael Kohlhaas [1810], trad. d'Armel Guerne et Robert Sctrick, Paris, Phébus, coll. «Verso », 1991. 
une instance impartiale (le seigneur est protégé au plus haut niveau), il entre en fureur, jette le masque du paisible marchand et se mue en impitoyable guerrier qui met toute l'Allemagne à feu et à sang pour quelques chevaux - ou plus exactement pour l'impossibilité de découper l'espace public et juridique dans lequel une plainte pourrait être énoncée et entendue à propos de cet incident - , est-ce bien raisonnable? À ce sujet, incidemment, il ne serait pas tout à fait inutile de se demander comment Ulysse (on parle ici de la fin de l'Odyssée, du massacre des prétendants, de la récupération du trône d'Ithaque et de sa place refroidie dans la couche de Pénélope) persiste à être aux yeux des anciens et aux nôtres aussi, un héros, jusqu'au bout, y compris au-delà de cette affreuse extermination, tandis que Kohlhaas, lui, nous apparaît comme une sorte de monstre ou de dément, et Chabert, nous le verrons, au bout du chemin, bien pire encore, un douceâtre martyr chrétien. Ulysse, en effet, n'est-il pas, en l'occurrence, celui qui, d'emblée, renonce à exposer le litige qui l'oppose aux prétendants sur un mode procédural, à se mettre en quête de l'introuvable tribunal qui le rétablira dans ses droits? Ulysse est cette figure éloignée, perdue pour nous dans les lointains du passé, qui n'a pas de temps à perdre à chercher les moyens de phraser autour du différend qui l'oppose à ses adversaires. Il ne s'en remet pas à l'impossible décision d'un juge, mais à celle de son arc. Il rétablit ses droits tout seul, parce que lui, il bande encore - le fameux arc, bien sûr. C'est une option, face au différend, face à l'échec de la mise en phrases de la plainte, face à la fâcheuse tendance des juges à se défiler ou de perdre les dossiers. Gardons-la en mémoire, ce qui nous changera un peu de notre propension croissante à nous installer vertueusement dans le rôle du chœur, c'est-à-dire des pleureuses et de leurs «hélas, trois fois hélas » dédiées sans fin à la mémoire des malheureuses victimes - je pense ici aux Perses d'Eschyle en premier lieu.

Il s'agit de poser assez abruptement cette question, histoire de prendre date à propos de cet enjeu qui est au cœur de préoccupations amplement partagées aujourd'hui: quels sont les présupposés cachés de l'évidence contemporaine selon laquelle nous avons à adopter sur tous les désastres présents et passés, les immenses et les infinitésimaux, le point de vue des victimes? Ce ralliement, conditionné par notre âme sensible davantage que par des choix philosophiques ou éthiques délibérés, est-il de nature à fonder le meilleur des récits possibles des désastres et la plus efficace des résistances possibles à ceux qui s'annoncent?

Revenons à Chabert. Sa rencontre avec l'avoué Derville produit une soudaine interruption de la malédiction qui le frappe: pour la première fois, quelqu'un l'écoute, prête foi à son récit, adopte son parti. L’homme de loi se déclare prêt à l'aider à faire valoir ses droits. Cet infléchissement du destin, Chabert le 
nomme un miracle: «les paroles du jeune avoué furent donc un miracle pour cet homme rebuté pendant dix années par sa femme, par la justice, par la création sociale entière». L'avoué fait les démarches nécessaires pour retrouver les documents qui prouvent son identité, une procédure s'engage en vue d'une conciliation avec son ancienne épouse, et dès l'instant où Chabert se trouve ainsi réinclus dans un espace juridique, qu'il peut faire valoir son point de vue dans un débat contradictoire, il connaît une véritable résurrection, on persiste dans le registre lazaréen. Aussi longtemps que sa plainte n'était pas entendue, reçue devant une instance de type judiciaire, Chabert demeurait en marge de l'humanité; il se décrit lui-même comme un «débris » de l'épopée napoléonienne, ballotté par les éléments dans cette sorte de tempête qu'est l'Histoire (Benjamin). Il évoque son errance de « débris curieux, après avoir ainsi roulé sur le globe comme roulent dans l'Océan les cailloux emportés d'un rivage à l'autre par les tempêtes ». Cette dérive ne se décrit pas seulement comme déclassement, mais bien comme perte d'humanité. Ce n'est pas seulement qu'il a perdu son nom, c'est aussi qu'il a perdu son visage, son apparence humaine : «Comment aurais-je pu intéresser une femme? J'avais une face de Requiem, j'étais vêtu comme un sans-culotte, je ressemblais plutôt à un Esquimau qu'à un Français, moi qui jadis passais pour le plus joli des muscadins, en 1799! Moi, Chabert, comte de l'Empire!» (CC, p. 54).

On voit bien ici le chemin qui conduit de l'impossibilité de se faire le narrateur d'un récit de catastrophe, de l'incapacité du narrateur à articuler son désastre propre à cette sorte de retour à la nature, au sauvage - «l'Esquimau ». Comment témoigner de son propre devenir-autre, quasi-animal?

Au reste, Chabert étant ainsi devenu «méconnaissable», selon ses propres termes, il va perdre le dernier des recours de type juridique, dans son effort pour prouver qu'il est bien celui qu'il dit être, le recours à un témoin, à défaut de preuves matérielles. Son compagnon d'armes, le maréchal des logis, qui a assisté à sa fausse mort sur le champ de bataille d'Eylau, qui, en 1814, l'a identifié après son retour à la vie, ne le reconnaît plus, à Paris, à l'issue de tant d'années d'errances et de tribulations. Quand Chabert retrouve sa trace et l'adjure de se porter garant de son identité, l'autre demeure sceptique et refuse de témoigner. Il y a tant d'imposteurs, d'usurpations d'identité en ces temps troublés, après toutes ces hécatombes, tous ces bouleversements!

À ce stade, donc, Chabert a touché le fond du calvaire infligé au survivant: il a perdu toute puissance sociale, a perdu son nom, ne peut rien faire valoir de ses états de service, il n'a plus figure humaine et est voué pour cette raison à la plus rigoureuse des solitudes. La fable imaginée par Balzac est tout entière guidée par 
cette intuition cruelle: les vivants ont horreur des survivants parce que ceux-ci ont séjourné trop longtemps du côté de la mort, trop près d'elle, et y ont perdu leur figure humaine. Le premier mouvement des vivants ordinaires à l'endroit des survivants n'est pas de compassion mais de vindicte. L'ancienne épouse de Chabert donne un corps et un nom à ce premier mouvement en ne ménageant aucun effort pour renvoyer ce spectre à son néant, pour, dit Balzac, «l'anéantir socialement» (CC, p. 99). Ce n'est que dans un deuxième temps que les vivants, épouvantés par ce qui se découvre d’abîme de méchanceté ontologique dans ce premier mouvement, vont s'adonner à cette piété victimophile sans rivage qui nous est devenue une seconde nature. Mais n'ayons garde de l'oublier : les années de l'immédiat après-guerre, dans un pays comme la France, pour ne rien dire de la Pologne, sont des années où l'antisémitisme est encore et toujours florissant et prospère, non pas comme si rien ne s'était passé, mais bien plutôt comme si, au fond, chaque survivant était de trop, coupable d'être revenu de l'enfer, suspect à ce titre.

Le second mouvement qui porte à se rallier à tous les récits de victimes, à raconter tous les désastres selon leur point de vue, n’est donc pas celui de la compassion ou de la pitié rousseauiste (souffrir avec), mais bien celui de ce que Nietzsche appelle la mauvaise conscience, affect réactif par excellence. Balzac écrit son Colonel Chabert en un temps, dans un topos où l'horreur du survivant, en tant que victime, peut encore se déployer et s'exposer assez tranquillement, parmi les vivants. Ce temps n'est plus le nôtre, semble-t-il.

Quoi qu'il en soit, Chabert, après avoir touché le fond, avoir connu l'épreuve dont, par excellence on ne peut témoigner, celle de la dégradation d'une vie qualifiée en vie nue, Chabert quitte la position du chasseur Gracchus de Kafka ${ }^{8}$, ce no man's land entre vie et mort, dès lors que l'entremise de l'avoué Derville lui permet d'accéder à un espace public, de renouer avec l'interlocution. Balzac décrit non sans une certaine ironie cette sorte de résurrection:

Le défunt arriva donc voituré dans un cabriolet fort propre. Il avait la tête couverte d'une perruque appropriée à sa physionomie, il était habillé de drap bleu, avait du linge blanc, et portait sous son gilet le sautoir rouge des grands officiers de la légion d'honneur. En reprenant les habitudes de l'aisance, il avait retrouvé son ancienne élégance martiale. (cC, p. 88)

8. Franz Kafka : «Le Chasseur Gracchus» [1917], dans La Muraille de Chine et autres récits, trad. Jean Carrive et Alexandre Vialatte, Paris, Éditions Gallimard, coll. «Folio», 1984. 
On a ici une illustration très concrète, imagée, de l'opposition grecque, réactivée par Arendt et Agamben9, entre zoe (vie) et bios (vie qualifiée). Une vie qualifiée, c'est une existence propre qui est dotée de la capacité à se présenter au monde et aux autres humains en exhibant les attributs d'une singularité, en exposant et en faisant valoir une distinction, des signes particuliers: ici, une décoration, du linge blanc (signe d'appartenance à la bonne société; la plèbe ne porte pas de linge blanc), une perruque seyante, une voiture. Autant de signes d'appartenance. À partir de là, tout peut s'enchainer: Chabert retrouve voix au chapitre, il est visible, il peut se présenter devant une instance arbitrale et obtenir réparation. Une fin morale se dessine, celle à laquelle nous aspirons tous, en présence des désastres et des violences extrêmes - la réadoption des victimes, la pratique de rites d'expiation ou de compensation appropriés, l'apaisement des douleurs, et, grâce à la somme de ces gestes, le retour à la normale. Mais ce n'est qu'un trompe-l'œil et tout l'art de Balzac est de nous conduire sur cette fausse piste (le différend réparé) pour mieux nous faire tomber dans l'embuscade et nous asséner son coup de massue philosophique - le différend ne se règle pas, le désastre ne se répare pas. Leçon antidialectique, qui laisse le négatif de l'histoire sans relève ni sauvetage. Chabert, au fond, n'aspirait, pour toute réparation, qu'à une chose: retrouver, sinon l'amour, du moins l'« estime » de son épouse, c'est-àdire entrer dans un jeu de reconnaissance mutuelle avec elle. Pouvoir se réjouir de ce qu'enfin, elle l'identifie en tant que vivant, comme ce qu'il n'a jamais cessé d'être. Il n'exige rien d'elle, si ce n'est qu'elle valide son retour en dignité humaine - la dignitas romaine, c'est d'abord un rang, d'où découle, ensuite, une valeur morale.

Mais nous l'avons vu, la comtesse Ferraud n'aspire qu'à une chose: que Chabert disparaisse - et elle manœuvre, elle intrigue sans relâche à cette fin. Lorsque enfin Chabert voit clair dans son jeu, il est anéanti: il renonce à se battre, abandonne toute la procédure, il se laisse retomber dans sa position acosmique. Bientôt arrêté, condamné comme vagabond, il est interné à l'hôpital de Bicêtre où il retombe en enfance. Derville qui a fini par retrouver sa piste lui rend visite et le trouve jouant au soldat, comme un enfant. Comme il le salue d'un «Bonjour colonel Chabert!», le vieillard répond: «Pas Chabert! pas Chabert! je me nomme Hyacynthe [...] Je ne suis plus un homme, je suis le numéro 164,

9. Voir, notamment: Hannah Arendt, Les Origines du totalitarisme. L'Impérialisme [1951], trad. Martine Leiris, Paris, Éditions du Seuil, coll. «Points», 1984, (chapitre 5: «Le déclin de l'État-nation et la fin des Droits de l'Homme») et Giorgio Agamben, Ce qui reste d'Auschwitz. 
septième salle» (CC, p. 113, je souligne). Sur le visage de ce numéro qui n'est plus un homme, dit Balzac, se lit «une anxiété peureuse, une crainte de vieillard et d'enfant».

Le jeune avoué lui glisse dans la main une pièce de vingt francs pour s'acheter du tabac et s'en va. Il l'entend qui psalmodie dans son dos, tandis qu'il s'éloigne «Feu des deux pièces! Vive Napoléon!» (CC, p. 113). Le souvenir de l'autre Histoire possible, celle d'une reprise de la grande épopée, n'est plus conservé que par un vieillard retombé en enfance.

Ou bien encore: ce que nous dit donc cette fin sarcastique et cruelle est explicite. Au temps de ce que Foucault appelle l'histoire massacrante ${ }^{10}$, temps inauguré pour lui par les carnages napoléoniens, précisément, l'extrême se donne à voir comme l'irréparable, l'irrelevable. Au moment précis où il s'avise de ce que l'amour de sa jeunesse ne désire rien d'autre que son retour dans la fosse commune d'Eylau (d'où il n'aurait jamais dû sortir, aux yeux des vivants), il renonce à parler, à présenter le tort, à espérer une réparation. Il se laisse doucement glisser à nouveau dans la fosse. C'est une sorte de suicide, entre stoïcisme, dit Balzac, et désespoir. C'est la certitude poignante que le survivant ne peut pas obtenir justice ni même rencontrer les conditions d'une réception publique de son récit d'épreuve. Et donc, il choisit cette ligne de fuite benjaminienne, si l'on veut, il retombe en enfance, il revient à l'enfance, il joue, lui l'ancien guerrier, aux petits soldats. Il descend doucement vers l'infime, le dérisoire, l'insignifiant, l'infra-humain, le quasi-animal, vers l'abject auquel l'a brièvement arraché Derville. Ulysse, lui, se redressait et accomplissait sa dernière geste de héros en faisant coïncider sur un mode terrible la figure du justicier (du droit) et celle de la guerre, de l'extermination, en construisant des machines de guerre qui étaient simultanément des machines à réparer les torts, voire à régler de vive force les différends.

Dans les deux cas, remarquons-le, les procédures régulières de la justice et de la réparation sont en panne. Et donc, on voit bien que face à la multiplication de ce genre de situation placé sous l'empire du différend, on a toujours deux solutions: soit adopter le jeu d'Ulysse, soit celui de Chabert. Dans tous les cas, contrairement à ceux qui s'en remettent en tout aux potentialités du langage, à nos facultés de prendre la parole ou de communiquer, il n'y a pas matière à

10. Michel Foucault: «Les meurtres qu'on raconte », dans Moi, Pierre Rivière, ayant égorgé ma mère, ma sœur et mon frère, un cas de parricide au XIX ${ }^{e}$ siècle, Paris, Éditions Gallimard, «Archives»,1973, p. 265 et sq. 
phraser. Il y a des corps qui entrent en action ou qui, au contraire, se laissent dépérir.

Irrésistiblement et par contraste, la nouvelle de Balzac fait penser à une autre œuvre notoire, le sinistre tableau du baron Antoine Gros intitulé Le champ de bataille d'Eylau (1808), typique document d'histoire de vainqueurs où l'on voit l'Empereur caracolant à la lisière des cadavres et des agonisants, un chromo que Benjamin avait peut-être en tête lorsqu' il écrivit, dans sa fameuse thèse VII : «Tous ceux qui jusqu'ici ont remporté la victoire participent à ce cortège triomphal où les maitres d'aujourd'hui marchent sur le corps des vaincus d'aujourd'hui [...]. Il n'est aucun document de culture qui ne soit aussi document de barbarie ${ }^{11} »$. Aux antipodes du document de barbarie fabriqué par le courtisan Gros, Le colonel Chabert se présente à nos yeux, en dépit de sa fin un peu sulpicienne, comme la contribution de Balzac à l'histoire des vaincus, c'est-à-dire, si l'on veut, à une histoire de la disparition. L'épopée napoléonienne n'y est pas vue du haut d'un cheval, de haut en bas, sous un angle où les cadavres des soldats ne sont qu'un entremêlement indistinct, mais bien du fond de la fosse commune, là où, dit Benjamin, même les morts ne sont pas en sécurité. Deux gestes s'opposent ici: celui de l'Empereur qui tend la main vers l'horizon indéfini de ses conquêtes, à la manière d'Alexandre, et celui du mort-vivant qui se hisse hors de la fosse en s'agrippant au bras raidi par le froid et la mort d'un de ses camarades massacrés. Le haut et le bas, toujours. Mais libre à vous d'inverser les positions, en disant, par exemple, comme le pamphlétaire Paul-Louis Courier, commentant, vers 1804, les aspirations impériales du général Bonaparte: «Il aspire à descendre : il veut se faire empereur ${ }^{12} »$.

11. Walter Benjamin, «Thèses sur la philosophie de l'histoire» [1940], dans [Euvres 2. Poésie et Révolution, trad. Maurice de Gandillac, Paris, Denoël, coll. «Les Lettres nouvelles", 1971, p. 281.

12. Paul-Louis Courier, «Lettre à M. N., à Plaisance» [1804], Pamphlets, Paris, Éditions Jean-Jacques Pauvert, coll. «Libertés», 1966. 